Pacific Journal of Mathematics

ON THE HAUSDORFF-YOUNG THEOREM FOR INTEGRAL 


\title{
ON THE HAUSDORFF-YOUNG THEOREM FOR INTEGRAL OPERATORS
}

\author{
BERNARD RUSSO
}

\begin{abstract}
A sharp inequality of Hausdorff-Young type is proved for integral operators. Applications are made in operator theory and in harmonic analysis on locally compact groups.
\end{abstract}

In recent work the author stated an inequality of Hausdorff-Young type for integral operators which proved to be useful in obtaining $L^{p}$ estimates on certain locally compact unimodular groups. The present paper is devoted to a closer analysis of that inequality together with some applications to operator theory and to $L^{p}$-Fourier analysis on locally compact groups.

In the first place, the proof given previously in [15 I], for the inequality is incomplete, so this paper will begin in $\$ 2$ with a correct proof of the inequality (Theorem 1). Also shown in $\$ 2$ is the nonexistence of extremal functions in a particular instance (Prop. 8). In $\$ 3$ the results of $\$ 2$ are applied to obtain estimates for the norm of the $L^{p}$-Fourier transform on certain unimodular groups. Here some of the machinery from [10] is used in the examples, one class of which (Prop. 13) does not depend on Theorem 1. This has happened before, see [15 I: §3]. For certain members of this class however, it is shown that a better estimate can be obtained using Theorem 1 (Prop. 15). In $\$ 4$ the study of Hausdorff-Young inequalities on nonunimodular groups is initiated. In view of the recent work on Plancherel formulas for nonunimodular groups such inequalities with constant 1 might be considered routine. It is shown here using Theorem 1, that the natural Hausdorff-Young inequality on the " $a x+b$ " group has a constant less than 1 (Prop. 19). In $\$ 5$ an operator valued analog of the Fourier transform on Abelian groups is introduced, which is motivated by preceding sections, and it is shown, using Theorem 1, that it behaves in some respects like the Fourier transform (Prop. 20).

\section{The Hausdorf-Young Theorem for integral} operators. Let $X$ be a $\sigma$-finite measure space, $k \in L^{2}(X \times X)$, and let $K$ be the integral operator with kernel $k$, i.e.,

$$
K f(x)=\int k(x, y) f(y) d y, \quad f \in L^{2}(X), \quad x \in X
$$


For $1 \leqq p, q<\infty$ the mixed norms (cf. [3]) are defined by

$$
\|k\|_{p, q}=\left(\int\left(\int|k(x, y)|^{p} d x\right)^{q / p} d y\right)^{1 / q}
$$

with the obvious meaning if $p$ or $q$ is $\infty$, and $k^{*}$ is defined by $k^{*}(x, y)=\overline{k(y, x)}$. For any bounded linear operator $T$ on $L^{2}(X)$

$$
\|T\|_{r}=\left\{\operatorname{tr}\left(T^{*} T\right)^{r / 2}\right\}^{1 / r}, \quad 1 \leqq r<\infty,
$$

and $\|T\|_{\infty}=$ the operator bound of $T$. Then $c_{p}$ denotes the set of operators $T$ with $\|T\|_{p}<\infty$.

THeOrem 1. Let $1<p<2, p^{\prime}=p /(p-1)$ and let $k \in L^{2}(X \times X)$ where $X$ is a $\sigma$-finite measure space. If $K$ is the integral operator with kernel $k$, then

$$
\|K\|_{p^{\prime}} \leqq\left(\|k\|_{p, p^{\prime}}\left\|k^{*}\right\|_{p, p^{\prime}}\right)^{1 / 2}
$$

The proof will be preceded by five lemmas, the first three of which are proved by standard measure theoretic techniques.

LEMMA 2. If $p, q \in[1, \infty)$ and $\|k\|_{p, q}<\infty$, then there exist simple functions $\left\{s_{n}\right\}$ on $X \times X$ (independent of $p$ and $q$ ) such that $\left\|k-s_{n}\right\|_{p, q} \rightarrow 0$ and $\left\|k^{*}-s_{n}^{*}\right\|_{p, q} \rightarrow 0$.

Lemma 3. Let $E$ be a measurable subset of $X \times X$ such that $\left\|\chi_{E}\right\|_{p, q}<\infty$ for some $p, q \in[1, \infty)$. Then

$$
\lim _{\substack{|F| \rightarrow 0 \\ F C E}}\left\|\chi_{F}\right\|_{p, q}=0
$$

where $|F|$ is the measure of $F$ and $\chi_{F}$ is the characteristic function of $F$.

LEMMA 4. For each measurable subset $E$ of $X \times X$ of finite measure and each $\delta>0$, there exists a set $F$ which is a finite disjoint union of measurable rectangles such that $|E \Delta F|<\delta$.

For the convenience of the reader it is remarked that the sequence $\left\{s_{n}\right\}$ in Lemma 2 is just the usual one [7: (11.35)], Lemma 3 is a consequence of dominated convergence, and Lemma 4 can be reduced to the case of a finite measure space whence the proof follows by showing that the collection of sets with the desired property is a monotone class. 
Lemma 5. Let $Z=X \times X$ and define $\varphi \in L^{2}(Z \times Z)$ by $\varphi(z, w)=$ $k\left(z_{1}, w_{1}\right) k^{*}\left(z_{2}, w_{2}\right)$ for $z=\left(z_{1}, z_{2}\right), w=\left(w_{1}, w_{2}\right) \in Z$. Let $\Phi$ be the integral operator on $L^{2}(Z)$ with kernel $\varphi$. Then

(1) $\|\varphi\|_{p, q}=\left\|\varphi^{*}\right\|_{p, q}=\|k\|_{p, q}\left\|k^{*}\right\|_{p, q}$ for $p, q \in[1, \infty]$.

(2) $\|\Phi\|_{r}=\|K\|_{r}^{2}$ for $1 \leqq r \leqq \infty$.

Proof. (1) is immediate from the definition of the mixed norms and (2) is immediate once it is noticed that $\Phi=K \otimes K^{*}$.

REMARK 6. If $K=\sum_{\imath} \lambda_{i} \xi_{l} \otimes \bar{\Psi}_{\iota}, \lambda_{\imath}>0,\left\{\xi_{l}\right\},\left\{\Psi_{\imath}\right\}$ ON sets in $L^{2}(X)$, is the "spectral" decomposition of $K$ in the sense of [16], then $\Phi=$ $\Sigma_{(b, j)} \lambda_{l} \lambda_{l}\left(\xi_{i} \times \Psi_{j}\right) \otimes \overline{\left(\Psi_{i} \times \xi_{j}\right)}$ is the "spectral" decomposition of $\Phi$.

The following crude estimate was known to the author in 1972 and appears in [14].

LEMMA 7. Let $k=\sum_{\imath=1}^{m} \alpha_{\imath} \chi_{A_{i} \times B_{i}}$ where $\alpha_{\imath} \in \mathbf{C}$ and $\left\{A_{i} \times B_{\imath}\right\}_{i=1}^{m}$ are pairwise disjoint measurable rectangles in $X \times X$. Then for $1<p<2$, $p^{\prime}=p /(p-1)$

$$
\|K\|_{p^{\prime}} \leqq 2^{1 / p^{\prime}} \max \left(\|k\|_{p, p^{\prime}},\left\|k^{*}\right\|_{p, p^{\prime}}\right)
$$

The proof of (5) is an adaptation of a standard interpolation argument inspired by [3: §7] and based on the validity of (4) at the endpoints $p=1,2$. The crudeness of the result and the necessary modifications are due to the circumstance that although $\|k\|_{1, \infty}$ (resp. $\left\|k^{*}\right\|_{1, \infty}$ ) and $\|k\|_{2,2}$ interpolate to $\|k\|_{p, p^{\prime}}$ (resp. $\left\|k^{*}\right\|_{p, p^{\prime}}$ ) it is problematical whether $\max \left(\|k\|_{1, \infty},\left\|k^{*}\right\|_{1, \infty}\right)$ and $\|k\|_{2,2}$ interpolate to $O\left(\max \left(\|k\|_{p, p^{\prime}},\left\|k^{*}\right\|_{p, p^{\prime}}\right)\right)$.

Proof of Lemma 7. Assume that $\|k\|_{p, p^{\prime}} \leqq 1$ and $\left\|k^{*}\right\|_{p, p^{\prime}} \leqq 1$. For $\xi \in \mathbf{C}$ define a function $k_{\xi}$ by the rule

$$
k_{\xi}(x, y)=|k(x, y)|^{(2-\xi) p / 2} \operatorname{sgn} k(x, y) M(x, y)^{-p+\xi\left(p^{\prime}+p\right) / 2}
$$

when $k(x, y) \neq 0$ and $k_{\xi}(x, y)=0$ if $k(x, y)=0$ where $M(x, y)=$ $\max \left(\|k(x, \cdot)\|_{p},\|k(\cdot, y)\|_{p}\right)$. The function $k_{\xi}$ is supported on a set of finite measure and bounded for $\xi$ in the strip $\Omega: 0 \leqq \operatorname{Re} \xi \leqq 1$. Thus $k_{\xi} \in L^{2}(X \times X)$ for $\xi \in \Omega$ and so defines an integral operator $K_{\xi}$ which is at least Hilbert Schmidt. Let $A$ be a finite rank operator on $L^{2}(X)$ with polar decomposition $A=V|A|$ and assume $\|A\|_{p} \leqq 1$. Set

$$
A_{\xi}=V|A|^{(2-\xi) p / 2} \quad \text { for } \quad \xi \in \mathbf{C}
$$


so that $A_{\xi}$ is Hilbert Schmidt and $K_{\xi} A_{\xi}$ is trace class for $\xi \in \Omega$. Thus $F(\xi)=\operatorname{tr}\left(K_{\xi} A_{\xi}\right)$ exists for $\xi \in \Omega$ and we have

$$
|F(\xi)| \leqq\left\|K_{\xi}\right\|_{p}\left\|A_{\xi}\right\|_{p}, \quad \xi \in \Omega, \quad 1 \leqq p \leqq \infty .
$$

It is easily verified that $F$ is analytic in the interior of $\Omega$ and continuous and bounded on $\Omega$. For $t$ real, $\left\|A_{u}\right\|_{1}=\|A\|_{p}^{p} \leqq 1 ;\left\|k_{u}\right\|_{1, x} \leqq 1$, and $\left\|\left(k_{u t}\right)^{*}\right\|_{1, \infty} \leqq 1$. Thus (7) with $p=1, \xi=i t$ and the case $p=1$ of (4) imply $|F(i t)| \leqq 1$. Again for $t$ real, $\left\|A_{1+u}\right\|_{2}^{2}=\|A\|_{p}^{p} \leqq 1$, and $\left\|k_{1+u t}\right\|_{2}^{2} \leqq 2$, the latter since

$$
\left|k_{1+u}\right|^{2}=|k|^{p} M^{p^{\prime}-p}, \quad\left\|k_{1+u t}\right\|_{2}^{2}=\left(\iint_{S}+\iint_{T}\right)\left|k_{1+u}\right|^{2} d x d y
$$

where $S=\left\{(x, y) \in X \times X:\|k(x, \cdot)\|_{p} \geqq\|k(\cdot, y)\|_{p}\right\}$ and $T=X \times X-S$, so that

$$
\begin{aligned}
& \left\|k_{1+u}\right\|_{2}^{2} \leqq \iint_{S}|k(x, y)|^{p}\|k(x, \cdot)\|_{p}^{p^{\prime}-p} d x d y \\
& +\iint_{T}|k(x, y)|^{p}\|k(\cdot, y)\|_{p}^{p^{\prime}-p} d x d y \\
& \leqq\left\|k^{*}\right\|_{p, p^{\prime}}+\|k\|_{p, p^{\prime}} \leqq 2 \text {. }
\end{aligned}
$$

Finally (7) with $p=2, \xi=1+i$ and the case $p=2$ of (4) imply $|F(1+i t)| \leqq \sqrt{2}$. By the three lines theorem $|F(s+i t)| \leqq(\sqrt{2})^{s}$ for every real $s, 0 \leqq s \leqq 1$. Putting $s=2 / p^{\prime}$ and $t=0$ results in $|\operatorname{tr}(K A)|=$ $\left|F\left(2 / p^{\prime}\right)\right| \leqq 2^{1 / p^{\prime}}$ so that $\|K\|_{p^{\prime}}=\sup \left\{|\operatorname{tr}(K A)|:\|A\|_{p} \leqq 1, A\right.$ finite rank $\} \leqq$ $2^{1 / p^{\prime}}$ and (5) is proved.

Proof of Theorem 1. By an approximation argument using Lemmas 2, 3, 4 (and described in [15-I: p. 301]) (5) holds for arbitrary $k \in L^{2}(X \times X)$. For a given $k$ define $X_{0}=X \times X, k_{0}=k$ and for $n \geqq 1$, $X_{n}=X_{n-1} \times X_{n-1}, \quad k_{n}(z, w)=k_{n-1}\left(z_{1}, w_{1}\right) k_{n-1}^{*}\left(z_{2}, w_{2}\right)$ for $z=\left(z_{1}, z_{2}\right)$ and $w=\left(w_{1}, w_{2}\right) \in X_{n}$. Let $K_{n}$ be the integral operator on $L^{2}\left(X_{n}\right)$ with kernel $k_{n}$. By Lemma 5

$$
\begin{array}{cc}
\left\|K_{n}\right\|_{p^{\prime}}=\left\|K_{n-1}\right\|_{p^{\prime}}^{2}, & n=1,2, \cdots \\
\left\|k_{n}\right\|_{p, p^{\prime}}=\left\|k_{n}^{*}\right\|_{p, p^{\prime}}=\left\|k_{n-1}\right\|_{p, p^{\prime}}\left\|k_{n-1}^{*}\right\|_{p, p^{\prime}} & n=1,2, \cdots
\end{array}
$$

and by Lemma 7

$$
\left\|K_{n}\right\|_{p^{\prime}} \leqq 2^{1 / p^{\prime}} \max \left(\left\|k_{n}\right\|_{p, p^{\prime}},\left\|k_{n}^{*}\right\|_{p, p^{\prime}}\right) \quad n=0,1,2, \cdots
$$


It follows that

$$
\|K\|_{p^{\prime}} \leqq\left(2^{1 / p^{\prime}}\right)^{2-n}\left(\|k\|_{p, p^{\prime}}\left\|k^{*}\right\|_{p, p^{\prime}}\right)^{1 / 2} \quad n=1,2, \cdots
$$

and (4) follows by letting $n \rightarrow \infty$.

For the first application consider kernels of the following sort: let $f$ and $g$ be measurable functions on $\mathbf{R}^{n}$ and let $k(x, y)=f(x-y) g(y)$ so that the corresponding operator $K$ has the form $K=L_{f} M_{g}, L_{f}=$ convolution by $f, M_{g}=$ multiplication by $g$. The following inequality, valid for $1<p<2,1 / p+1 / p^{\prime}=1$, can be obtained from Theorem 1 or by using a known interpolation theorem [11: Th. 3]:

$$
\left\|L_{f} M_{g}\right\|_{p^{\prime}} \leqq\|f\|_{p}\|g\|_{p^{\prime}}
$$

Although it is not known if 1 is the best constant in (8) the following result seems to indicate that it is not.

Proposition 8. If the two sides of (8) are equal and finite, then $f=0$ a.e., or $g=0$ a.e.

Proof. Note first that $\|k\|_{p, p^{\prime}}=\|f\|_{p}\|g\|_{p^{\prime}}$ and by a change of variable and Minkowski's integral inequality, that

$$
\left\|k^{*}\right\|_{p, p^{\prime}} \leqq\left(\int\left(\int|f(y)|^{p^{\prime}}|g(x-y)|^{p^{\prime}} d x\right)^{p / p^{\prime}} d y\right)^{1 / p}=\|k\|_{p, p^{\prime}}
$$

By assumption $\|K\|_{p^{\prime}}=\|k\|_{p, p^{\prime}}$ so by Theorem $1\|K\|_{p^{\prime}} \leqq\left\|k^{*}\right\|_{p, p^{\prime}}$ $\leqq\|k\|_{p, p^{\prime}}=\|K\|_{p^{\prime}}$ and so there is equality in (9), whence [5: p. 148] $|f(y)|^{p}|g(x-y)|^{p}=\varphi(x) \Psi(y)$ a.e. $(x, y)$. This forces $f=0$ a.e., or $g=0$ a.e.

Remark 9. Since it is not known if extremal functions exist in general or even in this example it cannot be asserted at this point that $\left\|\mathscr{F}_{p}\left(\Gamma_{3}\right)\right\|<A_{p}^{2}$.

3. Unimodular groups. In this section $L^{p}$ estimates are obtained for some examples of unimodular groups whose Plancherel formulas were derived in [10].

If $G$ is a locally compact separable group and $\omega$ is a multiplier on $G$, then the set of equivalence classes of irreducible $\omega$-representations of $G$ is denoted by $\hat{G}^{\omega}$. If the group $G(\bar{\omega})$ has a type I regular representation and if $G$ is unimodular then there is a measure $\mu=\mu_{G, \omega}$ on $\hat{G}^{\omega}$ such that 


$$
\int_{\hat{G}^{\omega}}\left\|\pi_{\xi}(f)\right\|_{2}^{2} d \mu(\xi)=\int_{G}|f(x)|^{2} d x, \quad f \in L^{1}(G) \cap L^{2}(G),
$$

where $\pi_{\xi} \in \xi$ for $\mu$-almost all $\xi$. This is the unimodular version of the projective Plancherel theorem of Kleppner and Lipsman [10 I: Th. 7.1]. As an immediate consequence of (10) and [12: Th. 3] one has:

Proposition 10. Let $G$ be a locally compact separable unimodular group with normalized multiplier $\omega$ such that $G(\bar{\omega})$ has a Type I regular representation. Then for $1<p<2$ there is a constant $\left\|\mathscr{F}_{p}(G, \omega)\right\| \leqq 1$ such that

$$
\left(\int_{\hat{G}^{\omega}}\left\|\pi_{\xi}(f)\right\|_{p^{\prime}}^{p^{\prime}} d \mu(\xi)\right)^{1 / p^{\prime}} \leqq\left\|\mathscr{F}_{p}(G, \omega)\right\|\left(\int_{G}|f(x)|^{p} d x\right)^{1 / p}
$$

for $f \in L^{p}(G)$, where $1 / p+1 / p^{\prime}=1$.

REMARK 11. It is natural as a generalization of [15] to try to improve the estimate $\left\|\mathscr{F}_{p}(G, \omega)\right\| \leqq 1$ as $G$ and $\omega$ vary. This question is not pursued here. For the example $G=\mathbf{R}^{2}$ and $\omega=\omega_{\gamma}$ presented in [10 I, p. 490] one can easily show, using Theorem 1 , that $\left\|\mathscr{F}_{p}\left(\mathbf{R}^{2}, \omega_{\gamma}\right)\right\| \leqq A_{p}$ $(<1)$. It is also clear that the exact value of $\left\|\mathscr{F}_{p}\left(\mathbf{R}^{2}, \omega_{\gamma}\right)\right\|$ can be obtained once $\left\|\mathscr{O}_{p}(\mathbf{R})\right\|$ is known (see $\$ 5$ ).

Proposition 12. Let $G$ be unimodular and of type I and separable and let $N$ be the center of $G$. Suppose that for all $\gamma \in N$, the group $(G / N)$ $\left(\bar{\omega}_{\gamma}\right)$ is of type I. Then

$$
\left\|\mathscr{F}_{p}(G)\right\| \leqq\left\|\mathscr{F}_{p}(N)\right\| \quad \text { for } \quad 1 \leqq p \leqq 2
$$

Proof. For groups $G$ satisfying the hypothesis the Plancherel formula is described in [10 I: Th. 8.1]. That and an interpolation argument as in Prop. 10 imply

$$
\|\hat{\varphi}\|_{p^{\prime}}=\left(\int_{\hat{N}} \int_{\widehat{(G / N) \omega_{\gamma}^{\omega_{\gamma}}}}\left\|\pi_{\gamma, \sigma}(\varphi)\right\|_{p^{\prime}}^{p^{\prime}} d \mu_{G / N, \bar{\omega}_{\gamma}}(\sigma) d \gamma\right)^{1 / p^{\prime}} \leqq\|\varphi\|_{p}
$$

for $\varphi \in L^{p}(G), 1<p<2,1 / p+1 / p=1, N=$ Cent $G$. Recall from [10 I] that $\pi_{\gamma, \sigma}=\gamma^{\prime} \otimes \sigma^{\prime \prime}$ where $\gamma^{\prime}$ is an $\omega_{\gamma}$-representation of $G$ which extends $\gamma \in \hat{N}$ and $\sigma^{\prime \prime}$ is the lift of $\sigma \in(\widehat{G / N})^{\omega_{\gamma}}$ to $G$ and $\omega_{\gamma}$ is the multiplier on $G / N$ corresponding to $\gamma$. It follows that 


$$
\begin{aligned}
\pi_{\gamma, \sigma}(\varphi) & =\int_{G} \varphi(g) \gamma^{\prime}(g) \sigma^{\prime \prime}(g) d g=\int_{G / N} \int_{N} \varphi(n g) \gamma^{\prime}(n g) \sigma^{\prime \prime}(n g) d n d \bar{g} \\
& =\int_{G / N} \int_{N} \varphi(n g) \overline{\omega_{\gamma}(n, g)} \gamma(n) \gamma^{\prime}(g) \sigma(\bar{g}) d n d \bar{g} \\
& =\sigma\left(\Psi_{\gamma}\right) \quad \text { where } \quad \Psi_{\gamma}(\bar{g})=\left[\varphi(\cdot g) \overline{\omega_{\gamma}(\cdot, g)} \gamma^{\prime}(g)\right]^{\wedge}(-\gamma) .
\end{aligned}
$$

Therefore

$$
\begin{aligned}
\|\hat{\varphi}\|_{p^{\prime}}^{p^{\prime}} & =\int_{\hat{N}} \int_{(\widehat{G / N})^{\dot{\omega}_{\gamma}}}\left\|\sigma\left(\Psi_{\gamma}\right)\right\|_{p^{\prime}}^{p^{\prime}} d \mu_{G / N, \bar{\omega}_{\gamma}}(\sigma) d \gamma \\
& \leqq \int_{\hat{N}}\left\|\Psi_{\gamma}\right\|_{p}^{p^{\prime}} d \gamma=\int_{\hat{N}}\left(\int_{G / N}\left|\Psi_{\gamma}(\bar{g})\right|^{p} d \bar{g}\right)^{p^{\prime} / p} d \gamma \\
& \leqq\left(\int_{G / N}\left(\int_{\hat{N}}\left|\Psi_{\gamma}(\bar{g})\right|^{p^{\prime}} d \gamma\right)^{p / p^{\prime}} d \bar{g}\right)^{p^{\prime} / p} \\
& \leqq\left(\int_{G / N}\left\|\mathscr{F}_{p}(N)\right\| \int_{N}\left|\varphi(n g) \overline{\bar{\omega}_{\gamma}(n, g)} \gamma^{\prime}(g)\right|^{p} d n d \bar{g}\right)^{p^{\prime} / p} \\
& =\left\|\mathscr{F}_{p}(N)\right\|^{p^{\prime}}\left(\int_{G / N} \int_{N}|\varphi(n g)|^{p} d n d \bar{g}\right)^{p^{\prime} / p}=\left\|\mathscr{F}_{p}(N)\right\|^{p^{\prime}}\|\varphi\|_{p}^{p^{\prime}} .
\end{aligned}
$$

This result is meaningful only if $\left\|\mathscr{F}_{p}(N)\right\|<1$, i.e., $N=$ Cent $G$ has no compact open subgroups, e.g., if $G$ is a connected simply connected real nilpotent group. It can also be applied to certain solvable groups e.g. the oscillator group, and to an example of a semi-direct product given in [10 II: $\$ 3$ (c)] which gives rise to the Weil representation. The estimates given by Prop. 12 for these examples are still crude. For example, for the Heisenberg group $\Gamma_{3}$ with one dimensional center it is already known that $\left\|\mathscr{F}_{p}\left(\Gamma_{3}\right)\right\| \leqq A_{p}^{2}<A_{p}=\left\|\mathscr{F}_{p}(\mathbf{R})\right\|$. Note that Theorem 1 was not used in the proof of Proposition 12. The next example satisfies the hypothesis of Proposition 12 but has a compact center. However a meaningful estimate can still be obtained by using Theorem 1.

EXAmPLE 13. Let $G_{1}=G / D$ where $G$ is the three dimensional Heisenberg group and $D$ is the subgroup

$$
D=\left\{\left(\begin{array}{ccc}
1 & 0 & 2 \pi n \\
0 & 1 & 0 \\
0 & 0 & 1
\end{array}\right): \quad n \in Z\right\}
$$


The Plancherel formula is given in [10 I: p. 514]. It reads

$$
\int_{G_{1}}\left|\varphi\left(g_{1}\right)\right|^{2} d g_{1}=\int_{\mathbf{R}^{2}}\left|\pi_{0, \sigma}(\varphi)\right|^{2} d \sigma+\sum_{n \neq 0}|n|\left\|\pi_{n}(\varphi)\right\|_{2}^{2}
$$

for $\varphi \in L^{1}\left(G_{1}\right) \cap L^{2}\left(G_{1}\right)$, where for $n \neq 0$ the kernel of the integral operator $\pi_{n}(\varphi)$ is $k_{n}(x, u)=\varphi(x-u, \cdot, \cdot)^{\wedge}(-n u,-n)$ (Fourier transform on $\mathbf{R} \times T)$ and $\pi_{0, \sigma}$ is just the character on $\mathbf{R}^{2}$ given by $\sigma \in \mathbf{R}^{2}$. Interpolating (13) yields

$$
\|\hat{\varphi}\|_{p^{\prime}}^{p^{\prime}}=\int_{\mathbf{R}^{2}}\left|\pi_{0, \sigma}(\varphi)\right|^{p^{\prime}} d \sigma+\sum_{n \neq 0}|n|\left\|\pi_{n}(\varphi)\right\|_{p^{\prime}}^{p^{\prime}}
$$

Now

$$
\begin{aligned}
\sum_{n \neq 0}|n|\left\|k_{n}\right\|_{p, p^{\prime}}^{p^{\prime}} & =\sum_{n \neq 0}|n| \int\left(\int\left|\varphi(x-u, \cdot, \cdot)^{\wedge}(-n u,-n)\right|^{p} d x\right)^{p^{\prime} / p} d u \\
& =\sum_{n \neq 0} \int_{\mathbf{R}}\left(\int_{\mathbf{R}}\left|\varphi(x, \cdot, \cdot)^{\wedge}(-v,-n)\right|^{p} d x\right)^{p^{\prime} / p} d v \\
& \leqq\left(\int_{\mathbf{R}}\left(\left.\sum_{n \neq 0} \int_{\mathbf{R}}\left|\varphi(x, \cdot, \cdot)^{\wedge}(-v,-n)\right|\right|^{p^{\prime}} d v\right)^{p / p^{\prime}} d x\right)^{p^{\prime / p}} \\
& =\left(\int_{\mathbf{R}}(F(x)-G(x))^{p^{/ p^{\prime}}} d x\right)^{p^{\prime / p}}=\|F-G\|_{p / p^{\prime}}
\end{aligned}
$$

where $F(x)=\left\|\varphi(x, \cdot, \cdot)^{\wedge}\right\|_{p^{\prime}}^{p^{\prime}}$ and $G(x)=\int_{\mathbf{R}}\left|\varphi(x, \cdot, \cdot)^{\wedge}(-v, 0)\right|^{p^{\prime}} d v$. But $F-G \geqq 0$ so by [7: (13.9)], $\|F\|_{p / p^{\prime}} \geqq\|F-G\|_{p / p^{\prime}}+\|G\|_{p / p^{\prime}}$. Thus

$$
\begin{aligned}
\sum_{n \neq 0}|n|\left\|k_{n}\right\|_{p, p^{\prime}}^{p^{\prime}} & \leqq\|F\|_{p / p^{\prime}}-\|G\|_{p / p^{\prime}}=\left(\int\left\|\varphi(x, \cdot, \cdot)^{\wedge}\right\|_{p^{\prime}}^{p} d x\right)^{p^{\prime} / p} \\
& -\left(\int_{\mathbf{R}}\left(\int_{R}\left|\varphi(x, \cdot, \cdot)^{\wedge}(-v, 0)\right|^{p^{\prime}} d v\right)^{p / p^{\prime}} d x\right)^{p^{\prime} / p} .
\end{aligned}
$$

Similarly

$$
\sum_{n \neq 0}|n|\left\|k_{n}^{*}\right\|_{p, p^{\prime}}^{p^{\prime}} \leqq\|F\|_{p / p^{\prime}}-\|G\|_{p / p^{\prime}}
$$




$$
\begin{aligned}
\|\hat{\varphi}\|_{p^{\prime}}^{p^{\prime}} \leqq & \int_{\mathbf{R}^{2}}\left|\pi_{0, \sigma}(\varphi)\right|^{p^{\prime}} d \sigma+\sum_{n \neq 0}|n|\left(\left\|k_{n}\right\|_{p, p^{\prime}}^{p^{\prime}}\left\|k_{n}^{*}\right\|_{p, p^{\prime}}^{p^{\prime}}\right)^{1 / 2} \\
\leqq & \int_{\mathbf{R}^{2}}\left|\pi_{0, \sigma}(\varphi)\right|^{p^{\prime}} d \sigma+\left(\sum|n|\left\|k_{n}\right\|_{p, p^{\prime}}^{p^{\prime}}\right)^{1 / 2}\left(\sum|n|\left\|k_{n}^{*}\right\|_{p, p^{\prime}}^{p^{\prime}}\right)^{1 / 2} \\
\leqq & \int_{\mathbf{R}^{2}}\left|\pi_{0, \sigma}(\varphi)\right|^{p^{\prime}} d \sigma+\|F\|_{p / p^{\prime}}-\|G\|_{p^{\prime} p^{\prime}} \\
\leqq & \int_{\mathbf{R}^{2}}\left|\pi_{0, \sigma}(\varphi)\right|^{p^{\prime}} d \sigma-\left(\int\left(\int\left|\varphi(x, \cdot, \cdot)^{\wedge}(-v, 0)\right|^{p^{\prime}} d v\right)^{p / p^{\prime}} d x\right)^{p^{\prime} / p} \\
& +\left\|\mathscr{F}_{p}(\mathbf{R} \times T)\right\|^{p^{\prime}}\|\varphi\|_{p}^{p^{\prime}} \\
\leqq & \left\|\mathscr{F}_{p}(\mathbf{R} \times T)\right\|^{p^{\prime}}\|\varphi\|_{p}^{p^{\prime}}
\end{aligned}
$$

since

$$
\begin{aligned}
\int_{\mathbf{R}^{2}}\left|\pi_{0, \sigma}(\varphi)\right|^{p^{\prime}} d \sigma & =\left.\iint\left|\pi_{0,(\omega, v)}(\varphi)\right|\right|^{p^{\prime}} d \omega d v \\
& =\iint|\hat{\varphi}(\omega, v, 0)|^{p^{\prime}} d \omega d v \\
& \leqq \int A_{p}^{p^{\prime}}\left(\left.\int\left|\varphi(x, \cdot, \cdot)^{\wedge}(v, 0)\right|\right|^{p} d x\right)^{p^{\prime / p}} d v \\
& \leqq\left(\int\left(\int\left|\varphi(x, \cdot, \cdot)^{\wedge}(v, 0)\right|^{p^{\prime}} d v\right)^{p / p^{\prime}} d x\right)^{p^{\prime} / p} .
\end{aligned}
$$

We conclude:

Proposition 14. For the group $G_{1}$ of the preceding example

$$
\left\|\mathscr{F}_{p}\left(G_{1}\right)\right\| \leqq\left\|\mathscr{F}_{p}(\mathbf{R})\right\|
$$

4. Nonunimodular groups. The result of this section is worked out for the two-dimensional solvable group $G$ known as the " $a x+b$ " group. It is clear that a calculation virtually identical to that given below will establish similar results for all of the solvable groups considered in [8: Ch. 4]. Before describing which realization of $G$ will be used it is noted that in this section the Fourier transform on $\mathbf{R}^{n}$ is defined by

$$
\hat{f}(y)=\int_{\mathbf{R}^{n}} e^{2 \pi i x y} f(x) d x
$$

and therefore 


$$
\left((-\Delta)^{\beta / 2} f\right)^{\wedge}(x)=(2 \pi|x|)^{\beta} \hat{f}(x)
$$

where $\Delta$ is the Laplacean.

Let $G=\mathbf{R} \times \mathbf{R}_{+}^{*}$ with group operation $(\lambda, s)(\mu, t)=(\lambda+s \mu, s t)$ right Haar measure $d_{R} x=d \lambda d s / s$, and modular function $\delta_{G}(\lambda, s)=1 / s$. The Plancherel formula for $G$ may be stated as follows [13: Th. 4] (cf. [10 I, 8, 9]): there is an operator $M$ in- $L^{2}(G)$ such that

$$
\|\hat{\Psi}\|_{2}^{2} \equiv\left\|\pi_{+}(\Psi)\right\|_{2}^{2}+\left\|\pi_{-}(\Psi)\right\|_{2}^{2}=\int_{G}|M \Psi(x)|^{2} d_{R} x \equiv\|M \Psi\|_{2}^{2}
$$

for $\Psi \in C_{c}^{\infty}(G)$. This $M$ is determined in Proposition 15 and $\left\{\pi_{+}, \pi_{-}\right\}$ are the two infinite dimensional members of the unitary dual $\hat{G}$ of $G$.

Proposition 15. Let $G$ be the " $a x+b$ " group, and let $1<p \leqq 2$, $1 / p+1 / p^{\prime}=1$. Then

$$
\|\hat{\Psi}\|_{p^{\prime}}=\left(\left\|\pi_{+}(\Psi)\right\|_{p^{\prime}}^{p^{\prime}}+\left\|\pi_{-}(\Psi)\right\|_{p^{\prime}}^{p^{\prime}}\right)^{1 / p^{\prime}} \leqq\left\|\mathscr{F}_{p}(\mathbf{R})\right\|\left\|M_{p} \Psi\right\|_{p}, \quad \Psi \in C_{c}^{\infty}(G)
$$

where $M_{p}=(2 \pi)^{1 / p^{\prime}}(-\Delta)^{-1 / 2 p^{\prime}} \otimes I$. Equality holds $($ for all $\Psi)$ for $p=2$.

Proof. The representation $\pi_{ \pm}$can be realized as acting on $L^{2}\left(\mathbf{R}_{+}^{*}, d s / s\right)$ by $\left(\pi_{ \pm}(\mu, t) g\right)(s)=e^{ \pm 2 \pi s \mu} g(s t), g \in L^{2}\left(\mathbf{R}_{+}^{*}, d s / s\right),(\mu, t) \in G$, $s \in \mathbf{R}_{+}^{*}$. Then $\pi_{ \pm}(\Psi)$ is an integral operator with kernel $k_{ \pm}(t, s)=$ $\Psi(\cdot, t)^{\wedge}\left( \pm s t^{-1}\right)$. Letting $\Sigma$ denote summation over $\{+,-\}$,

$$
\begin{aligned}
\|\hat{\Psi}\|_{p^{\prime}}^{p^{\prime}} & =\sum\left\|\pi_{ \pm}(\Psi)\right\|_{p^{\prime}}^{p^{\prime}} \leqq \sum\left(\left\|k_{ \pm}\right\|_{p, p^{\prime}}^{p^{\prime}}\left\|k_{ \pm}^{*}\right\|_{p, p^{\prime}}^{p^{\prime}}\right)^{1 / 2} \\
& \leqq\left(\sum\left\|k_{ \pm}\right\|_{p, p^{\prime}}^{p^{\prime}}\right)^{1 / 2}\left(\sum\left\|k_{ \pm}^{*}\right\|_{p, p^{\prime}}^{p^{\prime}}\right)^{1 / 2}
\end{aligned}
$$

Now

$$
\begin{aligned}
\sum\left\|k_{ \pm}\right\|_{p, p^{\prime}}^{p^{\prime}} & =\sum \int_{0}^{\infty}\left(\int_{0}^{\infty}\left|\Psi(\cdot, t)^{\wedge}\left( \pm s t^{-1}\right)\right|^{p} \frac{d t}{t}\right)^{p^{\prime} / p} \frac{d s}{s} \\
& \leqq\left(\int_{0}^{\infty}\left(\sum \int_{0}^{\infty}\left|\Psi(\cdot, t)^{\wedge}\left( \pm s t^{-1}\right)\right|^{p^{\prime}} \frac{d s}{s}\right)^{p / p^{\prime}} \frac{d t}{t}\right)^{p^{\prime / p}} \\
& =\left(\int^{\infty}\left(\sum \int_{0}^{\infty}\left|\Psi(\cdot, t)^{\wedge}( \pm s) s^{-1 / p^{\prime}}\right| p^{\prime} d s\right)^{p / p^{\prime}} \frac{d t}{t}\right)^{p^{\prime / p}} \\
& =\left(\int_{0}^{\infty}\left(\left.\int_{-\infty}^{\infty}\left|\Psi(\cdot, t)^{\wedge}(\lambda)\right| \lambda\right|^{-1 / p^{\prime}} \mid p^{\prime} d \lambda\right)^{p / p^{\prime}} \frac{d t}{t}\right)^{p^{\prime} / p} \\
& =\left(\int_{0}^{\infty}\left(\int_{-\infty}^{\infty}\left|(2 \pi)^{1 / p^{\prime}}\left((-\Delta)^{-1 / 2 p^{\prime}} \Psi(\cdot, t)\right)^{\wedge}(\lambda)\right|^{p^{\prime}} d \lambda\right)^{p / p^{\prime}} \frac{d t}{t}\right)^{p^{\prime} / p}
\end{aligned}
$$




$$
\begin{aligned}
& \leqq\left(\int_{0}^{\infty} A_{p}^{p}\left\|(2 \pi)^{1 / p^{\prime}}(-\Delta)^{-1 / 2 p^{\prime}} \Psi(\cdot, t)\right\|_{p}^{p} \frac{d t}{t}\right)^{p^{\prime} / p} \\
& =A_{p}^{p^{\prime}}\left\|M_{p} \Psi\right\|_{p}^{p^{\prime}}
\end{aligned}
$$

The same inequality holds for $\Sigma\left\|k_{ \pm}^{*}\right\|_{p, p^{\prime}}^{p^{\prime}}$ and the result follows.

5. Analog of the Fourier transform. Let $G$ be a locally compact Abelian group with dual group $\hat{G}$. For a measurable function $F$ on $\hat{G} \times G$ consider the kernel $k_{F}, k_{F}(\alpha, \beta)=F(\alpha-\beta, \cdot)^{\wedge}(\beta), \alpha, \beta \in \hat{G}$ and the corresponding integral operator $K_{F}$ defined by $K_{F} \varphi(\beta)=$ $\int_{\hat{G}} k_{F}(\alpha, \beta) \varphi(\beta) d \beta$. Using the estimates $\left\|K_{F}\right\|_{\infty} \leqq\|F\|_{1}$ and $\left\|K_{F}\right\|_{2}=\|F\|_{2}$ and the general theory of interpolation one obtains a mapping $\hat{O}_{p}(G): L^{p}(\hat{G} \times G) \rightarrow c_{p^{\prime}}\left(L^{2}(\hat{G})\right)$, where $1<p<2,1 / p+1 / p^{\prime}=1$, of norm $\leqq 1:\left\|K_{F}\right\|_{p^{\prime}} \leqq\|F\|_{p}$.

This type of kernel appears in the Fourier decomposition of the Heisenberg group, see $\$ 2$ (cf. [4: \$2], [15 II: §2]).

Proposition 16. Let $G$ be a locally compact Abelian group and let $1<p<2$. Then

(1) $\left\|\mathscr{O}_{p}(G)\right\| \leqq\left\|\mathscr{F}_{p}(G)\right\|$

(2) $\left\|\mathcal{O}_{p}(G)\right\|=1$ if and only if $G$ has a compact open subgroup.

(3) If $\left\|K_{F}\right\|_{p^{\prime}}=\left\|\mathscr{F}_{p}(\mathbf{R})\right\|\|F\|_{p}$ and $p^{\prime}$ is an even integer then $F=0$ a.e. $(G=\mathbf{R})$.

Proof. (1) Using Minkowski's integral inequality and changing variables one obtains that $\left\|k_{F}\right\|_{p, p^{\prime}}$ and $\left\|k_{F}^{*}\right\|_{p, p^{\prime}}$ are both $\leqq\left\|\mathscr{F}_{p}(G)\right\|\|F\|_{p}$ so (1) follows from Theorem 1. (2) If $\left\|O_{p}(G)\right\|=1$ then by (1), $\left\|\mathscr{F}_{p}(G)\right\|=1$ so $G$ has a compact open subgroup by [6: $\left.\$ 43\right]$ or [15 I: Th. 2]. Conversely if $G$ has a compact open subgroup $H$, then setting $F(\alpha, x)=c \chi_{H} \perp(\alpha) \chi_{H}(x)$ where $c$ is the measure of $H$ one finds that $\left\|K_{F}\right\|_{p^{\prime}}=\|F\|_{p^{*}}$ (3) The assumption implies that $\left\|k_{F}\right\|_{p, p^{\prime}}=\left\|k_{F}^{*}\right\|_{p, p^{\prime}}=$ $\left\|\mathscr{F}_{p}(\mathbf{R})\right\|\|F\|_{p}$ and hence that $F(\alpha, \cdot)$ is extremal in $L^{p}(\mathbf{R})$ for a.e. $\alpha$ and that $\left|F(\alpha, \cdot)^{\wedge}(\beta)\right|^{p}=\Phi(\alpha) \Psi(\beta)$. Thus

$$
F(\alpha, \beta)=c(\alpha) \exp \left\{-a(\alpha) \beta^{2}+i b(\alpha) \beta\right\}
$$

where $c(\alpha) \in \mathbf{C}, a(\alpha)>0$ and $b(\alpha) \in \mathbf{R}$. One finds that

$$
F(\alpha, \cdot)^{\wedge}(\beta)=\text { const } c(\alpha) /(a(\alpha))^{1 / 2} \times \exp \left\{-(b(\alpha)-\beta)^{2} / 4 a(\alpha)\right\}
$$


and and it follows from the above conditions that $a(\alpha)$ and $b(\alpha)$ do not depend on $\alpha$. Thus $F(\alpha, \beta)$ is of the form $f(\alpha) g(\beta), g$ Gaussian, so that $K_{F}=L_{f} M_{\hat{g}} \quad$ and $\quad\left\|\mathscr{F}_{p}(\mathbf{R})\right\|\|F\|_{p}=\left\|\mathscr{F}_{p}(\mathbf{R})\right\|\|f\|_{p}\|g\|_{p}=\|f\|_{p}\|\hat{g}\|_{p^{\prime}} \geqq$ $\left\|L_{f} M_{\hat{g}}\right\|_{p^{\prime}}=\left\|K_{F}\right\|_{p^{\prime}}$. By Proposition $8 f=0$ or $\hat{g}=0$ a.e. so $F=0$ a.e.

Remark 17. The property (3) in Proposition 16 indicates but does not prove that the numbers $\mathscr{F}_{p}(G)$ and $\mathscr{O}_{p}(G)$ are either distinct or both equal to 1.

REMARK 18. The behavior of $\mathscr{O}_{p}$ on products is not as simple as that of $\mathscr{F}_{p}$. In particular the computation of $\left\|\mathscr{O}_{p}\left(\mathbf{R}^{n}\right)\right\|$ is not automatically reducible to that of $\left\|\mathscr{O}_{p}(\mathbf{R})\right\|$ (cf. [2: Lemma 2]).

\section{REFERENCES}

1. K. I. Babenko, An inequality in the theory of Fourier integrals, Izv. Akad. Nauk. SSSR Ser. Mat., 25 (1961), 531-542. English Transl., Amer. Math. Soc. Transl. (2) 44, 115-128.

2. William Beckner, Inequalities in Fourier analysis, Ann. of Math., 102 (1975), 159-182.

3. A. Benedek and R. Panzone, The spaces $L^{P}$ with mixed norm, Duke Math. J., 28 (1961), 301-324.

4. Jacques Dixmier, Sur les représentations unitaires des groupes de Lie nilpotents. VI, Canad. J. Math., 12 (1960), 324-352.

5. G. H. Hardy, J. E. Littlewood, and G. Polya, Inequalities, Cambridge Univ. Press, 1964.

6. E. Hewitt and K. A. Ross, Abstract harmonic analysis. Vol. II, Springer-Verlag, Berlin and New York, 1970.

7. E. Hewitt and K. Stromberg, Real and abstract analysis, Springer-Verlag, Berlin and New York, 1969. MR 43 \# 428.

8. Frederick W. Keene, Square integrable representations of Iwasawa subgroups of a semi-simple Lie group, Doctoral dissertation, Univ. of Calif., Berkeley, 1974.

9. Idriss Khalil, Sur l'analyse harmonique du groupe affine de la droite, Stud. Math., 51 (1974), 139-167.

10. Adam Kleppner and Ronald L. Lipsman, The Plancherel formula for group extensions, I. Ann. Sci. École Norm. Sup., (4) 5 (1972), 459-516.; II, same journal 6 (1973) 103-132.

11. Ray A. Kunze, $L_{p}$-Fourier transforms on locally compact unimodular groups, Trans. Amer. Math. Soc., 89 (1958), 519-540.

12. Ray A. Kunze and Elias M. Stein, Uniformly bounded representations and harmonic analysis of the $2 \times 2$ real unimodular group, Amer. J. Math., 82 (1960), 1-62.

13. Lajos Pukanzsky, Unitary representations of solvable Lie groups, Ann. Scı. École Norm. Sup., (4) 4 (1971), 457-608.

14. Bernard Russo, Algebre di operatori e gruppi localmente compatti, mimeographed notes, University of Genoa, 1972.

15. The norm of the $L^{p}$-Fourier transform on unimodular groups, Trans. Amer. Math. Soc., 192 (1974), 293-305. II, Canad. J. Math., 28 (1976), 1121-1131. 
16. Robert Schatten, Norm ideals of completely continuous operators, Springer-Verlag, 1960.

17. Elias M. Stein, Singular integrals and differentiability properties of functions, Princeton Univ. Press, Princeton, N. J., 1970.

Received January 13, 1976 and in revised form May 5, 1976.

UNIVERSITY OF CALIFORNIA

IRVINE, CA 92717 




\section{Pacific Journal of Mathematics}

Vol. 68, No. 1

March, 1977

Richard Julian Bagby, On $L^{p}, L^{q}$ multipliers of Fourier transforms . .......

Robert Beauwens and Jean-Jacques Van Binnebeek, Convergence theorems in

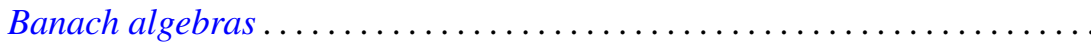

James Cyril Becker, Skew linear vector fields on spheres in the stable

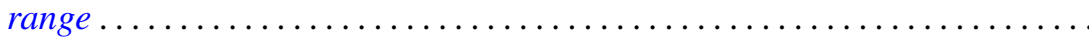

Michael James Beeson, Continuity and comprehension in intuitionistic formal

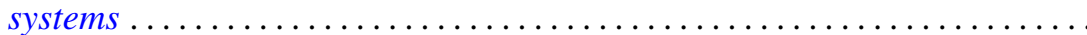

James K. Deveney, Generalized primitive elements for transcendental field

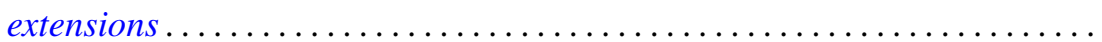

Samuel S. Feder, Samuel Carlos Gitler and K. Y. Lam, Composition properties

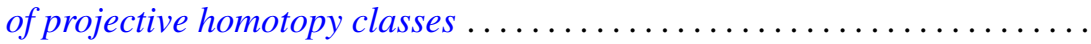

Nathan Jacob Fine, Tensor products of function rings under composition ......

Benno Fuchssteiner, Iterations and fixpoints . . . . . . . . . . . . . .

Wolfgang H. Heil, On punctured balls in manifolds

Shigeru Itoh, A random fixed point theorem for a multivalued contraction

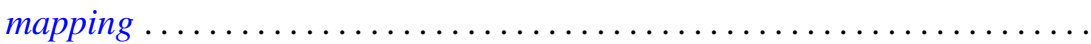

Nicolas P. Jewell, Continuity of module and higher derivations . . . . . . ......

Roger Dale Konyndyk, Residually central wreath products . . . . . . . . . . .

Linda M. Lesniak and John A. Roberts, On Ramsey theory and graphical

parameters.

Vo Thanh Liem, Some cellular subsets of the spheres.

Dieter Lutz, A perturbation theorem for spectral operators

P. H. Maserick, Moments of measures on convex bodies ... . . .

Stephen Joseph McAdam, Unmixed 2-dimensional local domains . .

D. B. McAlister and Norman R. Reilly, E-unitary covers for inverse semigroups...

William H. Meeks, III and Julie Patrusky, Representing codimension-one

homology classes by embedded submanifolds . . .

Premalata Mohapatro, Generalised quasi-Nörlund summability . .

Takahiko Nakazi, Superalgebras of weak-*Dirichlet algebras .

Catherine Louise Olsen, Norms of compact perturbations of operators .

William Henry Ruckle, Absolutely divergent series and isomorphism of

subspaces. II.

Bernard Russo, On the Hausdorff-Young theorem for integral operators .

Arthur Argyle Sagle and J. R. Schumi, Anti-commutative algebras and

homogeneous spaces with multiplications ............

Robert Evert Stong, Stiefel-Whitney classes of manifolds .

D. Suryanarayana, On a theorem of Apostol concerning Möbius functions of

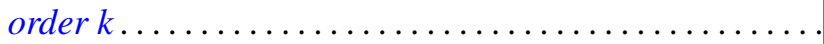

Yoshio Tanaka, On closedness of $C$ - and $C^{*}$-embeddings . . 